\title{
PACKING MEASURE AS A GAUGE VARIATION
}

\author{
G. A. EDGAR
}

(Communicated by Andrew M. Bruckner)

\begin{abstract}
Meinershagen noted that (in the line) the fractal packing measure of Tricot and Taylor can be considered to be a Henstock-Thomson gauge variation ("method III") for an appropriate choice of derivation basis and set function. We show that this point of view remains interesting in a general metric space.
\end{abstract}

\section{INTRODUCTION}

The best-known fractal measure is the Hausdorff measure. Tricot and Taylor $[13,10]$ propose the use of an additional fractal measure known as the packing measure. Fractals should be sets with some weak regularity properties, such as self-similarity. Taylor proposes that the term "fractal" should mean a set where the Hausdorff and packing measures both yield the same fractal dimension. A good reference for the packing measure is [9]. For packing measure in general metric spaces, see $[2,4]$.

Henstock [6] defined a family of "variation" set functions. Thomson [11] clarified the definition, calling it "method III" (following Munroe's methods I and II). In [12], he notes that if the variation is computed starting from the set function $(\operatorname{diam} B)^{s}$, where $s>0$ is a given positive number, then the resulting variation is an outer measure with the properties appropriate for an $s$-dimensional fractal measure. Thomson asked whether (in the line $\mathbf{R}$ ) this variation is the Hausdorff outer measure. Meinershagen [8] answered that this measure (the "symmetric derivation basis measure" computed with the set function $\left.(\operatorname{diam} B)^{s}\right)$ is the packing measure, not the Hausdorff measure. Meinershagen did her computations on the line $\mathbf{R}$. In this paper we show that in other metric spaces as well, the packing measure is a Henstock-Thomson gauge variation.

I will discuss "covering measure" and "Hausdorff measure" from this point of view in a subsequent paper.

\section{PACKing Measure}

1A. Tricot's definition. We begin with the definition of the packing measure $($ see $[13,10,9])$. Let $(S, \rho)$ be a metric space. For $x \in S$ and $r>0$, the open

Received by the editors December 8, 1992.

1991 Mathematics Subject Classification. Primary 28A80; Secondary 26A39.

Key words and phrases. Fractal measure, packing measure, gauge integral, Henstock variation, derivation basis. 
ball is

$$
B_{r}(x)=\{y \in S: \rho(y, x)<r\} .
$$

The diameter of a set $E \subseteq S$ is

$$
\operatorname{diam} E=\sup \{\rho(x, y): x, y \in E\} .
$$

Let $A \subseteq S$ be a set. A centered-ball packing of $A$ is a countable disjoint collection of open balls with center in $A$ :

$$
\left\{B_{r_{1}}\left(x_{1}\right), B_{r_{2}}\left(x_{2}\right), \cdots\right\},
$$

where $x_{i} \in A$ and $B_{r_{i}}\left(x_{i}\right) \cap B_{r_{j}}\left(x_{j}\right)=\varnothing$ for $i \neq j$.

(1.1) Definition. Let $s$ be a positive number. For $\varepsilon>0$, define

$$
\mathscr{P}_{\varepsilon}^{s}(A)=\sup \sum_{i}\left(\operatorname{diam} B_{r_{i}}\left(x_{i}\right)\right)^{s},
$$

where the supremum is over all packings of $A$ by centered balls with diameter $\leq \varepsilon$. [Of course, in Euclidean space diam $B_{r}(x)=2 r$, but in a general metric space this need not be true.] The s-dimensional packing pre-measure of $A$ is

$$
\widetilde{\mathscr{P}^{s}}(A)=\lim _{\varepsilon \rightarrow 0} \mathscr{P}_{\varepsilon}^{s}(A) \text {. }
$$

The s-dimensional packing outer measure is the outer measure $\mathscr{P} s$ defined from the set function $\widetilde{\mathscr{P}^{s}}$ by method I. That is,

$$
\mathscr{P}^{s}(A)=\inf \left\{\sum_{D \in \mathscr{D}} \widetilde{\mathscr{P}}^{s}(D): \mathscr{D} \text { is a countable cover of } A\right\} .
$$

Then $\mathscr{P}^{s}$ is a metric outer measure on $S$.

The definition is a bit awkward to use, because of the "method I" step added on the end. But the set function $\widetilde{\mathscr{P} s}$ is not countably subadditive, so this final step is needed to obtain a genuine outer measure.

The following are a few observations about the definition:

(1.2) Finite packings suffice. That is,

$$
\mathscr{P}_{\varepsilon}^{s}(A)=\sup \sum_{i}\left(\operatorname{diam} B_{r_{i}}\left(x_{i}\right)\right)^{s},
$$

where the supremum is over all finite packings of $A$ by centered balls with diameter $\leq \varepsilon$.

(1.3) An open ball $B_{r}(x)$ is the increasing union of a sequence $\bar{B}_{r-1 / n}(x)$ of closed balls, and $\operatorname{diam} \bar{B}_{r-1 / n}(x) \rightarrow \operatorname{diam} B_{r}(x)$. In many metric spaces, $\operatorname{diam} B_{r}(x)=\operatorname{diam} \bar{B}_{r}(x)$. If that is true, then the same value of $\widetilde{\mathscr{P}^{s}}$ is obtained if we use packings by closed balls instead of open balls. From a remark of Haase [4], it seems that this equality is false in general.

(1.4) If $A \subseteq S$ is a set, and $\bar{A}$ is the closure of the set, then $\widetilde{\mathscr{P}^{s}}(A)=\widetilde{\mathscr{P} s}(\bar{A})$. Indeed, on the one hand, any centered-ball packing of $A$ is also a centered-ball packing of $\bar{A}$, so $\widetilde{\mathscr{P} s}(A) \leq \widetilde{\mathscr{P} s}(\bar{A})$. On the other hand, if $B_{r}(x)$ is a ball with $x \in \bar{A}$, then there is a point $x^{\prime} \in A$ as close as we like to $x$ and a radius $r^{\prime}=r-\rho\left(x^{\prime}, x\right)$ so that $B_{r^{\prime}}\left(x^{\prime}\right) \subseteq B_{r}(x)$ and diam $B_{r^{\prime}}\left(x^{\prime}\right)$ is as close as we like to diam $B_{r}(x)$. Thus, given a centered-ball packing $\left\{B_{r_{i}}\left(x_{i}\right)\right\}$ of $\bar{A}$, we may 
choose a centered-ball packing $\left\{B_{r_{i}^{\prime}}\left(x_{i}^{\prime}\right)\right\}$ of $A$ with $\sum\left(\operatorname{diam} B_{r_{i}^{\prime}}\left(x_{i}^{\prime}\right)\right)^{s}$ as close as we like to $\sum\left(\operatorname{diam} B_{r_{i}}\left(x_{i}\right)\right)^{s}$. So $\widetilde{\mathscr{P}^{s}}(A)=\widetilde{\mathscr{P} s}(\bar{A})$.

(1.5) Since $\widetilde{\mathscr{P} s}(A)=\widetilde{\mathscr{P} s}(\bar{A})$, in the definition

$$
\mathscr{P}^{s}(A)=\inf \left\{\sum_{D \in \mathscr{D}} \widetilde{\mathscr{P}}^{s}(D): \mathscr{D} \text { is a countable cover of } A\right\}
$$

of $\mathscr{P s}$, it is enough to use covers $\mathscr{D}$ consisting of closed sets. Because of this fact, for any set $E \subseteq S$, there is a Borel set $A$ (in fact an $F_{\sigma \delta}$ ) with $A \supseteq E$ and $\mathscr{P}^{s}(A)=\mathscr{P}^{s}(E)$.

(1.6) If $E_{n}$ is an increasing sequence of (possibly nonmeasurable) sets, then we have $\lim _{n} \mathscr{P}^{s}\left(E_{n}\right)=\mathscr{P} s\left(\bigcup E_{n}\right)$. Indeed, for each $E_{n}$ choose a Borel set $A_{n} \supseteq E_{n}$ with the same outer measure, then consider $\bigcup_{n} A_{n}$.

(1.7) Given a set $A$, there is a critical value $s_{0} \in[0, \infty]$ such that

$$
\mathscr{P}^{s}(A)=\left\{\begin{aligned}
\infty & \text { for all } s \text { with } s<s_{0}, \\
0 & \text { for all } s \text { with } s>s_{0} .
\end{aligned}\right.
$$

This critical value is the packing dimension of the set $A$, which will be written $\operatorname{Dim} A=s_{0}$. At least in Euclidean space, the packing dimension coincides with the metrische Dimensionszahl of Wegmann [14, §3.3].

1B. Henstock's variation. The variation can be defined for a general "derivation basis" (Henstock [6]). Thomson [11] calls the construction "method III". Here we will use only the "centered ball" basis, so reference to the basis will be supressed from our notation and terminology.

Let $(S, \rho)$ be a metric space. A constituent is a pair $(B, x)$, where $x \in S$ is a point and $B$ is an open ball $B_{r}(x)$ with center $x$ and positive radius $r$. In many metric spaces, the center $x$ is uniquely determined by the ball $B$, but this fails in some metric spaces (such as ultrametric spaces), so the intended center is included as the second component of the constituent.

Let $E \subseteq S$. A packing of $E$ is a countable set

$$
\pi=\left\{\left(B_{1}, x_{1}\right),\left(B_{2}, x_{2}\right), \cdots\right\}
$$

of constituents with $x_{i} \in E$ for all $i$ and $B_{i} \cap B_{j}=\varnothing$ for $i \neq j$. The term packing by itself means a packing of the whole space $S$. A gauge on $E$ is a function $\Delta: E \rightarrow(0, \infty)$. If $\Delta$ is a gauge for $E$ and $\pi$ is a packing of $E$, then we say $\pi$ is $\Delta$-fine iff $\operatorname{diam} B \leq \Delta(x)$ for all constituents $(B, x)$ of $\pi$.

(1.8) Definition. Let $s$ be a positive number. For a gauge $\Delta$, define

$$
\mathscr{P}_{\Delta}^{s}(A)=\sup \sum_{(B, x) \in \pi}(\operatorname{diam} B)^{s},
$$

where the supremum is over all $\Delta$-fine packings $\pi$ of $A$. As $\Delta$ decreases toward 0 , the value $\mathscr{P}_{\Delta}^{s}(A)$ decreases. For the limit, write:

$$
\mathscr{P}_{\bullet}^{s}(A)=\inf _{\Delta} \mathscr{P}_{\Delta}^{s}(A) \text {. }
$$

The positive constants $\varepsilon$ are among the gauges, and if $\Delta(x)=\varepsilon$ for all $x$, then the notation $\mathscr{P}_{\Delta}^{s}$ is consistent with the previous notation $\mathscr{P}_{\varepsilon}^{s}$.

This time we get an outer measure without an extra "method I" step at the end. This is a special case of the general theory of [11], but we will prove it here because of its central importance. 
(1.9) Theorem. The set function $\mathscr{P}_{\bullet}$ is a metric outer measure.

Proof. The only packing of the empty set is the empty packing, so $\mathscr{P}_{\Delta}^{s}(\varnothing)=0$ for all $\Delta$, and $\mathscr{P}_{\bullet}^{s}(\varnothing)=0$.

If $E \subseteq F$, any packing of $E$ is also a packing of $F$, so $\mathscr{P}_{\Delta}^{s}(E) \leq \mathscr{P}_{\Delta}^{s}(F)$ for all $\Delta$, and thus $\mathscr{P}_{\bullet}^{s}(E) \leq \mathscr{P}_{\bullet}^{s}(F)$.

Suppose $E=\bigcup_{n=1}^{\infty} E_{n}$. We must show that $\mathscr{P}_{\bullet}^{s}(E) \leq \sum_{n} \mathscr{P}_{\bullet}^{s}\left(E_{n}\right)$. If the right-hand side is $\infty$, there is nothing to prove, so suppose it is finite. Fix $\varepsilon>0$. For each $n$, choose a gauge $\Delta_{n}$ on $E_{n}$ so that $\mathscr{P}_{\Delta_{n}}\left(E_{n}\right) \leq \mathscr{P}_{\bullet}\left(E_{n}\right)+\varepsilon / 2^{n}$. Define $\Delta$ by: $\Delta(x)=\Delta_{n}(x)$ if $x \in E_{n} \backslash \bigcup_{i=1}^{n-1} E_{i}$. Then $\Delta$ is a gauge on $E$. Let $\pi$ be a $\Delta$-fine packing of $E$. Then for each $n$,

$$
\pi_{n}=\left\{(B, x) \in \pi: x \in E_{n} \backslash \bigcup_{i=1}^{n-1} E_{i}\right\}
$$

is a $\Delta_{n}$-fine packing of $E_{n}$. So

$$
\begin{aligned}
\sum_{(B, x) \in \pi}(\operatorname{diam} B)^{s} & =\sum_{n} \sum_{(B, x) \in \pi_{n}}(\operatorname{diam} B)^{s} \\
& \leq \sum_{n} \mathscr{P}_{\Delta_{n}}^{s}\left(E_{n}\right) \leq \varepsilon+\sum_{n} \mathscr{P}_{\bullet}^{s}\left(E_{n}\right) .
\end{aligned}
$$

Therefore $\mathscr{P}_{\bullet}^{s}(E) \leq \mathscr{P}_{\Delta}^{s}(E) \leq \varepsilon+\sum \mathscr{P}_{\bullet}^{s}\left(E_{n}\right)$. Let $\varepsilon \rightarrow 0$ to obtain $\mathscr{P}_{\bullet}^{s}(E) \leq$ $\sum \mathscr{P} s_{\bullet}\left(E_{n}\right)$.

Suppose $E, F \subseteq S$ have positive separation, $\operatorname{dist}(E, F)=\varepsilon>0$. Let $\Delta$ be any gauge with $\Delta(x) \leq \varepsilon / 2$. Then the constituents of any $\Delta$-fine packing of $E$ are disjoint from the constituents of any $\Delta$-fine packing of $F$. So the union of the two packings is a $\Delta$-fine packing of $E \cup F$. Therefore $\mathscr{P}_{\Delta}^{s}(E \cup F)=$ $\mathscr{P}_{\Delta}^{s}(E)+\mathscr{P}_{\Delta}^{s}(F)$. This is true for all sufficiently small gauges, so $\mathscr{P}_{\bullet}^{s}(E \cup F)=$ $\mathscr{P ^ { s }}(E)+\mathscr{\mathscr { P } _ { \bullet }}(F)$.

Now in fact, the variation outer measure $\mathscr{P}_{\bullet}^{s}$ is just the packing outer measure $\mathscr{P}^{s}$; the notation $\mathscr{P}_{\bullet}^{s}$ was introduced for temporary use.

(1.10) Theorem. For any $E \subseteq S$ and $s>0$, we have $\mathscr{P}_{\bullet}(E)=\mathscr{P}^{s}(E)$.

Proof. The positive constants $\varepsilon$ are among the gauges, so

$$
\widetilde{\mathscr{P}^{s}}(E)=\inf _{\varepsilon} \mathscr{P}_{\varepsilon}^{s}(E) \geq \inf _{\Delta} \mathscr{P}_{\Delta}^{s}(E)=\mathscr{P}_{\bullet}(E)
$$

If $E \subseteq \bigcup_{n} E_{n}$, then $\mathscr{P}_{\bullet}^{s}(E) \leq \mathscr{P}_{\bullet}^{s}\left(\bigcup E_{n}\right) \leq \sum_{n} \mathscr{P}_{\bullet}^{s}\left(E_{n}\right) \leq \sum_{n} \widetilde{\mathscr{P} s}\left(E_{n}\right)$. Take the infimum over all such covers to conclude that $\mathscr{P}_{\bullet} s(E) \leq \mathscr{P}^{s}(E)$.

On the other hand, suppose $\Delta$ is a gauge on a set $E$. For each positive integer $n$ let

$$
E_{n}=\left\{x \in E: \Delta(x) \geq \frac{1}{n}\right\} .
$$

The sets $E_{n}$ increase to $E$. Now for each $n$,

$$
\mathscr{P}_{\Delta}^{s}(E) \geq \mathscr{P}_{\Delta}^{s}\left(E_{n}\right) \geq \mathscr{P}_{1 / n}^{s}\left(E_{n}\right) \geq \widetilde{\mathscr{P}}^{s}\left(E_{n}\right) \geq \mathscr{P}^{s}\left(E_{n}\right)
$$

So by $(1.6)$ we have $\mathscr{P}_{\Delta}^{s}(E) \geq \mathscr{P}^{s}(E)$. This is true for all gauges $\Delta$, so $\mathscr{P}_{\bullet}^{s}(E) \geq$ $\mathscr{P} s(E)$. 


\section{Gauge InTEgral}

If $f$ is a nonnegative Borel function, then the integral of $f$ may be computed as a variation.

(2.1) Definition. Let $h$ be a nonnegative real-valued constituent function; that is, for each constituent $(B, x)$, let $h(B, x) \in[0, \infty)$. If $\Delta$ is a gauge, write

$$
V_{\Delta}(h)=\sup \sum_{(B, x) \in \pi} h(B, x),
$$

where the supremum is over all $\Delta$-fine packings $\pi$ of $S$. (When $\Delta$ decreases, $V_{\Delta}(h)$ decreases.) The variation of $h$ is

$$
V(h)=\inf V_{\Delta}(h),
$$

where the infimum is over all gauges $\Delta$. When the constituent function $h$ is of the special form

$$
h(B, x)=f(x)(\operatorname{diam} B)^{s},
$$

for some nonnegative point-function $f: S \rightarrow[0, \infty)$, we will write $V_{\Delta}^{s}(f)=$ $V_{\Delta}(h)$ and $V^{s}(f)=V(h)$. We will call $V^{s}(f)$ the s-variation of $f$.

The following is proved in the same way as Theorem (1.9), above. Thomson calls $\mu$ a "method III" outer measure. We write $\mathbf{1}_{A}$ for the indicator function of a set $A$, and $h \mathbf{1}_{A}$ for the constituent function

$$
\left(h \mathbf{1}_{A}\right)(B, x)=h(B, x) \mathbf{1}_{A}(x) .
$$

(2.2) Theorem. Let $h$ be a nonnegative real-valued constituent function. Then $\mu$ defined by $\mu(A)=V\left(h \mathbf{1}_{A}\right)$ for all $A \subseteq S$ is a metric outer measure on $S$.

For special $h$ of the form $f(x)(\operatorname{diam} B)^{s}$, the variation is the integral with respect to the packing measure $\mathscr{P} s$.

(2.3) Proposition. Let $s>0$, and let $f$ be a nonnegative real-valued Borel function on $S$. Then

$$
V^{s}(f)=\int_{S} f(x) \mathscr{P}^{s}(d x) .
$$

Proof. First, by the respective definitions, if $A \subseteq S$ we have $V_{\Delta}^{s}\left(\mathbf{1}_{A}\right)=\mathscr{P}_{\Delta}^{s}(A)$ and $V^{s}\left(\mathbf{1}_{A}\right)=\mathscr{P}^{s}(A)$. Clearly, if $a \geq 0$ is a constant and $f \geq 0$ is a function, then $V^{s}(a f)=a V^{s}(f)$.

Now both $\mathscr{P} s$ and $V^{s}\left(f \mathbf{1}_{A}\right)$ are metric outer measures, so Borel sets are measurable. If $A_{n}$ are disjoint Borel sets and $a_{n} \geq 0$ are constants, then the simple function $f=\sum a_{n} \mathbf{1}_{A_{n}}$ satisfies $V^{s}(f)=\int_{S} f(x) \mathscr{P}^{s}(d x)$.

Finally, if $f$ is a nonnegative Borel function, then there is a sequence $f_{n}$ of nonnegative Borel measurable simple functions that increases to $f$. If $c<1$, then the sets

$$
E_{n}=\left\{x: f_{n}(x) \geq c f(x)\right\}
$$

increase to $S$. But $V^{s}\left(f_{n}\right) \geq c V^{s}\left(f 1_{E_{n}}\right)$, so $\lim _{n} V^{s}\left(f_{n}\right) \geq c V^{s}(f)$. Let $c \rightarrow 1$ to conclude that $V^{s}\left(f_{n}\right) \rightarrow V^{s}(f)$. Therefore we have

$$
V^{s}(f)=\lim _{n} V^{s}\left(f_{n}\right)=\lim _{n} \int_{S} f_{n}(x) \mathscr{P}^{s}(d x)=\int_{S} f(x) \mathscr{P}^{s}(d x),
$$

as required. 
Let $A \subseteq S$ be a set. A centered Vitali cover of $A$ is a set $\beta$ of constituents such that for every $x \in A$ and every $\varepsilon>0$, there is $(B, x) \in \beta$ with $\operatorname{diam} B \leq$ $\varepsilon$. A Borel measure $\mu$ has the strong Vitali property iff for any Borel set $A$ with $\mu(A)<\infty$ and any centered Vitali cover $\beta$ of $A$, there is a packing $\pi \subseteq \beta$ with

$$
\mu\left(A \backslash \bigcup_{(B, x) \in \pi} B\right)=0 .
$$

In many metric spaces, every Borel measure has the strong Vitali property. Besicovitch [1] proved this for Euclidean space (see also [5, p. 114]).

Let $\mu$ be a finite Borel measure on the metric space $S$. For any $x \in S$, the lower s-density of $\mu$ at $x$ is

$$
\underline{D}_{\mu}^{s}(x)=\liminf _{r \rightarrow 0} \frac{\mu\left(B_{r}(x)\right)}{\left(\operatorname{diam} B_{r}(x)\right)^{s}} .
$$

In this definition, the same lower density is obtained if we use closed balls rather than open balls; and the lim inf is unchanged if we remove any countable set of $r>0$. Saint Raymond and Tricot [9] prove that, in Euclidean space, if $\mathscr{P} s(E)<\infty$, then

$$
\mathscr{P}^{s}(E) \inf _{x \in E} \underline{D}_{\mu}^{s}(x) \leq \mu(E) \leq \mathscr{P}^{s}(E) \sup _{x \in E} \underline{D}_{\mu}^{s}(x) .
$$

The following is a strengthened form of this result.

(2.4) Theorem. Let $\mu$ be a finite Borel measure on the metric space $S$. For any Borel set $E \subseteq S$, we have

$$
\mu(E) \geq \int_{E} \underline{D}_{\mu}^{s}(x) \mathscr{P}^{s}(d x) .
$$

Equality holds if $\mu$ has the strong Vitali property, $\mathscr{P} s(E)<\infty$, and $\underline{D}_{\mu}^{s}(x)<\infty$ on $E$.

Proof. Let $E$ be a Borel set. Let $U \supseteq E$ be an open neighborhood of it, and let $c<1$ be a positive constant. For each $x \in E$, there is $\Delta(x)>0$ so that

$$
\Delta(x)<\operatorname{dist}(x, S \backslash U)
$$

and

$$
\frac{\mu\left(B_{r}(x)\right)}{\left(\operatorname{diam} B_{r}(x)\right)^{s}} \geq c \underline{D}_{\mu}^{s}(x) \quad \text { for all } r<\Delta(x) .
$$

So $\Delta$ is a gauge on $E$. If $\pi$ is a $\Delta$-fine packing of $E$, then

$$
\sum_{(B, x) \in \pi} \underline{D}_{\mu}^{s}(x)(\operatorname{diam} B)^{s} \leq \frac{1}{c} \sum_{(B, x) \in \pi} \mu(B) \leq \frac{1}{c} \mu(U) .
$$

Therefore $V^{s}\left(\underline{D}_{\mu}^{s} \mathbf{1}_{E}\right) \leq V_{\Delta}^{s}\left(\underline{D}_{\mu}^{s} \mathbf{1}_{E}\right) \leq(1 / c) \mu(U)$. Take the infimum over all open $U \supseteq E$ and all $c<1$ to obtain $V^{s}\left(\underline{D}_{\mu}^{s} \mathbf{1}_{E}\right) \leq \mu(E)$. But $\underline{D}_{\mu}^{s}$ is a Borel function, so this means

$$
\int_{E} \underline{D}_{\mu}^{s}(x) \mathscr{P}^{s}(d x) \leq \mu(E)
$$


Now suppose $\mu$ has the strong Vitali property and let $E$ be a Borel set with $\mathscr{P}^{s}(E)<\infty$. Let $\Delta$ be a gauge on $E$ such that $\mathscr{P}_{\Delta}^{s}(E)<\infty$ and let $\varepsilon>0$. Then

$$
\left\{(B, x) \text { constituent }: x \in E, \operatorname{diam} B \leq \Delta(x), \frac{\mu(B)}{(\operatorname{diam} B)^{s}} \leq \underline{D}_{\mu}^{s}(x)+\varepsilon\right\}
$$

is a centered Vitali cover of $E$. So we may choose from it a packing $\pi$ of $E$ with

$$
\mu\left(E \backslash \bigcup_{(B, x) \in \pi} B\right)=0 .
$$

Then we have

$$
\mu(E) \leq \sum_{(B, x) \in \pi} \mu(B) \leq \sum_{(B, x) \in \pi}\left(\underline{D}_{\mu}^{s}(x)+\varepsilon\right)(\operatorname{diam} B)^{s} \leq V_{\Delta}^{s}\left(\underline{D}_{\mu}^{s} \mathbf{1}_{E}\right)+\varepsilon_{\mathscr{P}_{\Delta}^{s}}(E) .
$$

Take the infimum over $\Delta$ and $\varepsilon$ to obtain $\mu(E) \leq V^{s}\left(\underline{D}_{\mu}^{s} \mathbf{1}_{E}\right)$.

Consider the example where $S=[0,1], \mu$ is Lebesgue measure on $[0,1]$, and $0<s<1$. Then $\underline{D}_{\mu}^{s}(x)=0$ for all $x$, but $\mu(S)>0$. This shows that the hypothesis $\mathscr{P}^{s}(E)<\infty$ cannot be dropped in the theorem.

\section{Questions}

The point of view exploited here suggests other questions.

What fractal measures will we get if we compute the Henstock gauge variation, using these same constituent functions, but other common differentiation bases? For example:

(3.1) Pairs $(B, x)$, where $B$ is an open ball and $x \in B$ (but possibly not the center).

(3.2) Pairs $(B, x)$, where $B$ is an open ball, and $x \in S$ is a point, possibly not in $B$ at all; " $\Delta$-fine" means that $B \subseteq B_{\Delta(x)}(x)$ (McShane basis).

(3.3) In Euclidean space, pairs $(B, x)$ where $B$ is a cube with sides parallel to the axes.

Hundreds of other bases can be found in [5].

C. Cutler [2] argues that the "radius packing" measure is superior to the "diameter packing" measure that has been used here.

(3.4) Carry out this study for the "radius packing" measure. This should be done by replacing the set-function $B_{r}(x) \mapsto\left(\operatorname{diam} B_{r}(x)\right)^{s}$ with the set-function $B_{r}(x) \mapsto(2 r)^{s}$.

\section{REFERENCES}

1. A. S. Besicovitch, $A$ general form of the covering principle and relative differentiation of additive functions, Proc. Cambridge Philos. Soc. 41 (1945), 103-110.

2. C. Cutler, The density theorem and Hausdorff inequality for packing measure in general metric spaces, Illinois J. Math. (to appear).

3. G. A. Edgar, Measure, topology, and fractal geometry, Springer-Verlag, New York, 1990.

4. H. Haase, The packing theorem and packing measure, Math. Nachr. 146 (1990), 77-84.

5. C. A. Hayes and C. Y. Pauc, Derivation and martingales, Springer-Verlag, New York, 1970. 
6. R. Henstock, Generalized integrals of vector-valued functions, Proc. London Math. Soc. 19 (1969), 509-536.

7. __ The general theory of integration, Clarendon Press, Oxford, 1991.

8. S. Meinershagen, The symmetric derivation basis measure and the packing measure, Proc. Amer. Math. Soc. 103 (1988), 813-814.

9. X. Saint Raymond and C. Tricot, Packing regularity of sets in n-space, Math. Proc. Cambridge Philos. Soc. 103 (1988), 133-145.

10. S. J. Taylor and C. Tricot, Packing measure, and its evaluation for a Brownian path, Trans. Amer. Math. Soc. 288 (1985), 679-699.

11. B. S. Thomson, Construction of measures in metric spaces, J. London Math. Soc. 14 (1976), 21-24.

12. __ Derivation bases on the real line (I), Real Anal. Exchange 8 (1982), 67-207.

13. C. Tricot, Two definitions of fractional dimension, Math. Proc. Cambridge Philos. Soc. 91 (1982), 57-74.

14. H. Wegmann, Die Hausdorff-Dimension von kartesischen Produkten metrischer Räume, J. Reine Angew. Math. 246 (1971), 46-75.

Department of Mathematics, The Ohio State University, Columbus, Ohio 43210

E-mail address: edgarOmath.ohio-state.edu 